\title{
iSense: App to Identify Linguistic Biomarkers of Mood Disorders
}

\section{Divya Nori}

\author{
Milton High School, Georgia
}

\begin{abstract}
The prevalence of mood disorders has increased rapidly over the past decade, especially in teens. The nature of these disorders is such that most symptoms are in a person's mind making it harder to diagnose, leading to a mental health epidemic which is difficult to control. This study proposes a mobile application (iSense) powered by an Artificial Intelligence model to identify linguistic biomarkers of mood disorders in a teen's outgoing SMS messages and send notifications to a parent. The Twitter API was used to create a dataset of over 800 individuals whose language use indicates a mood disorder, based on the DSM-5 Diagnostic Criteria for depressive disorders, and individuals with a healthy mental state. Millions of tweets were collected from these users and filtered to 73,944 tweets based on tweet length (over 50 characters) and dropping retweets. After conducting linguistic analysis, the tweets were used to train/test a generalized linear lasso model (hyperplane-based approach), gradient boosting machine (tree-based approach), and feed-forward multilayer perceptron (neural network-based approach). These models were then compared on several measures such as F1 Score $(\max 0.717)$ and accuracy $(\max 86 \%)$. Other features such as notification preferences (parent interface) and a voice-enabled chatbot (child interface) were implemented in the mobile application. iSense has the potential to enable early detection of mood disorders and at-risk individuals, providing a viable solution to combat the increase in suicide cases.
\end{abstract}

\section{Introduction}

Every 100 minutes, another teen takes their life, often with no perceivable warning. Suicide is the third- leading cause of death in young people age 15 to 19 , and the key to reducing its prevalence lies in detecting its top risk factor: mood disorders. A mood disorder is a category of mental illness in which the underlying problem primarily affects a person's persistent emotional state.

The prevalence of mood disorders in the United States among adolescents is $14.3 \%$, and $11.2 \%$ suffer from severe impairment, which is often a precursor to possible suicidal behavior [1].

\begin{tabular}{|c|c|c|}
\hline \multicolumn{2}{|c|}{ Demographic } & Percent \\
\hline \multicolumn{2}{|c|}{ Overall } & 14.3 \\
\hline \multicolumn{2}{|c|}{ With Severe Impairment } & 11.2 \\
\hline \multirow{2}{*}{ Sex } & Female & 18.3 \\
\cline { 2 - 3 } & Male & 10.5 \\
\hline \multirow{2}{*}{ Age } & $\mathbf{1 3 - 1 4}$ & 10.5 \\
\cline { 2 - 3 } & $\mathbf{1 5 - 1 6}$ & 15.5 \\
\cline { 2 - 3 } & $\mathbf{1 7 - 1 8}$ & 18.1 \\
\cline { 2 - 3 } & & \\
\hline
\end{tabular}

Over the past few years, several initiatives have attempted to detect suicidal tendencies in teens. In 2016, school guidance counselors in most states implemented mandated suicide awareness programs in high schools and assured students that their door is open. However, the likelihood that a teen who is seriously considering suicide would speak to a counselor is slim. Other initiatives recently taken in schools are suicide risk screenings, at-risk student referrals, and crisis emergency responses [2].

In the context of these challenges, this study examines and develops a novel solution based on the implications of these attempted solutions. As several past studies have found, social media and technology usage has risen exponentially, and has been repeatedly linked with the increased isolation and mental health concerns among adolescents. The research presented here analyzes social media as a 
potential tool to detect mood disorders. As people turn to platforms such as Twitter to express their daily thoughts, social media becomes a source of data through which mood disorders can be detected. By training an Artificial Intelligence (AI)-based model on this data, signs of a possible mood disorder can efficiently be detected.

However, social media alone cannot effectively solve this problem. While teens are more likely to reveal how they feel on social media when compared to in a counselor's office, detecting mood disorders on a public site is still not very effective. It is more likely that a teen will reveal how they truly feel to a friend through SMS. In addition, an efficient framework to take the information extracted from the AI-based model and notify a parent or trusted adult has not been developed.

This study aims to investigate the following question: Can a mathematical model identify and quantify the relationship between a healthy individual's language use in comparison to that of an individual with a mood disorder?

The hypothesis pursued is, if a healthy individual's language use is compared to that of an individual with a mood disorder, then there exists a difference in terms of linguistic biomarkers which can be identified by a mathematical model.

The main contributions in this paper are as follows:

(1) A Python script utilizing the Twitter API is developed to create a comprehensive case and control cohorts with over 70,000 tweets resulting in 1,854 features.

(2) A Generalized Linear Model, Gradient Boosting Machine, and Multilayer Perceptron

(3) iSense, an Android application backed by the model, is developed to detect mood disorders in a teen's SMS messages and notify a parent or trusted adult. Other features designed to aid in this goal are also incorporated. While the technology does not restrict the age of the users, it has been with a teen-parent audience in mind.
The solution proposed in this study combats the problems posed by the previously attempted solutions and has the potential to reduce the prevalence of teen suicide.

\section{Background Literature}

Several bodies of work in the intersection of clinical and cognitive psychology help more specifically identify possible correlations between linguistic structures and mood disorders. Mood disorders can be classified into two broad groups: unipolar and bipolar disorders [7]. Unipolar disorders, also known as depressive disorders, include Disruptive Mood Dysregulation Disorder, Persistent Depressive Disorder, and Major Depressive Disorder. Disruptive Mood Dysregulation Disorder is a childhood/adolescent condition characterized by extreme anger or irritability. Persistent Depressive Disorder is a mild but long-term form of depression. For an individual to be diagnosed with Major Depressive Disorder, they must have had at least one depressive episode in their life. Bipolar disorders are characterized by periods of depression alternating with periods of mania, including Bipolar I, Bipolar II, and Cyclothymic Disorder. An individual diagnosed with Bipolar I must have had at least one manic episode, often with periods of depression. Bipolar II is similar to Bipolar I, but manic episodes never reach a full high. Cyclothymic Disorder is characterized by emotional highs and lows, but it is not as severe as Bipolar Disorder. This study focuses on the presence of depression and the its top risk factors (untreated anxiety, substance abuse, low selfesteem, etc.), whether it be part of a unipolar depressive disorder or the depressed period of a bipolar disorder [8].

In 2015, researchers from the Department of Clinical Psychology and Psychotherapy at the University of Duisburg-Essen analyzed self-referent word usage ("me", "myself", "I") as an indicator of self-focused attention in relation to depression and anxiety. Selffocused attention is a cognitive bias that is closely related to depression, and this group attempted to characterize this relationship as disorder-specific or as a trans-diagnostic phenomenon. They used a sample of twenty-nine diagnosed individuals and 
thirty healthy individuals and found that in patients with Major Depressive Disorder, first-person singular pronouns were commonly used during the recall of a negative memory. In contrast, in healthy individuals as well as individuals with anorexia nervosa, first-person pronoun use increased when recalling a positive memory [4].

Researchers from the Center for Clinical Research in Neuropsychiatry attempted to identify deviations from typical word usage in individuals diagnosed with depression in 2018. They used a sample of 201 subjects to distinguish between natural language constructs associated with mild depression, normal sadness, and euthymic state. Linguistic indicators were found to distinguish between these states, including the use of pronouns (analyzed through lexico-grammatical test), syntactic elements (single clause vs multi clause), and rhetorical figures (similes, metaphors, etc.). Like the previous study, they found that individuals diagnosed with mild depression used a greater number of pronouns within a self-focused context. [5].

In the context of identifying these linguistic constructs on social media, in 2013, Microsoft Research conducted a study in which they attempted to measure behavioral attributes such as social engagement, emotion, language use, ego network, mentions of medication, etc. on Twitter. They then leveraged this information to build a statistical classifier which can estimate risk of depression. They found that unigrams such as "anxiety", "withdrawal", and "severe" indicated symptoms of depressive disorders. Trends concerning first person pronoun usage, swear word use, and frequency of depression terms were present in tweets classified as cases. They classifier accuracy of $70 \%$.

Another approach to predicting depression from language-based emotion dynamics was taken by the Monash Institute of Cognitive and Clinical Neurosciences. They conducted a longitudinal analysis of Facebook and Twitter status updates in May 2018. As previous studies primarily focused on linking average metric values, this group aimed to rely on dynamic measures of emotional volatility. Depression severity ratings and status updates were collected from 29 Facebook users and 49 Twitter users using the app MoodPrism. They showed that negative word usage was highly correlated to the severity of depression. Their findings suggest that negative emotion word instability may be a sensitive measure of time-structured variability, and may prove to effectively predict depression on a social media platform [6].

While the literature summarized above is useful in helping to define the terms of this study, previous research does not provide a sample size that is wide enough to effectively predict mood disorders on a real-world scale. The lack of data suggests that further research is needed to determine the linguistic biomarkers indicative of these disorders. Additionally, a cost-effective, efficient, and accurate solution that can be used in the real world has not been developed.

\section{Methods}

This study was designed as a case-control study, cases exhibiting the existence of a mood disorder and controls exhibiting a healthy mental state. Different exposures including linguistic structure and specific biomarkers were analyzed to better detect mood disorders and maximize the app's effectiveness. The following methods were used:

\section{Data Collection}

The Tweepy Twitter API was used to collect ground truth train and test data. Tweepy is an open-source library that enables Python to communicate with the Twitter Platform. A script which utilizes these capabilities was run for seven days, and over 1.5 million tweets and the corresponding twitter handles were collected in real time. Other than the filtering of retweets, no parameters were set and a large random sample of tweets was gathered.

After collecting this data from the Twitter database, each tweet was manually classified as indicative of anxiety/depression, indicative of a healthy emotional state, or irrelevant (tweeted by a company, automated account, etc.). Simply filtering on words such as "depression" or "depressed" would not be effective because the presence of those words alone 
does not indicate the presence of a depressive disorders (ex. tweets from mental health awareness accounts). To ensure that the tweet was truly from an individual suffering from a mood disorder, the basic Twitter interface was utilized to search the corresponding user timeline and determine whether the tweet could be classified as a case. Over 800 case and control individuals were identified based on the DSM-5 Criteria:

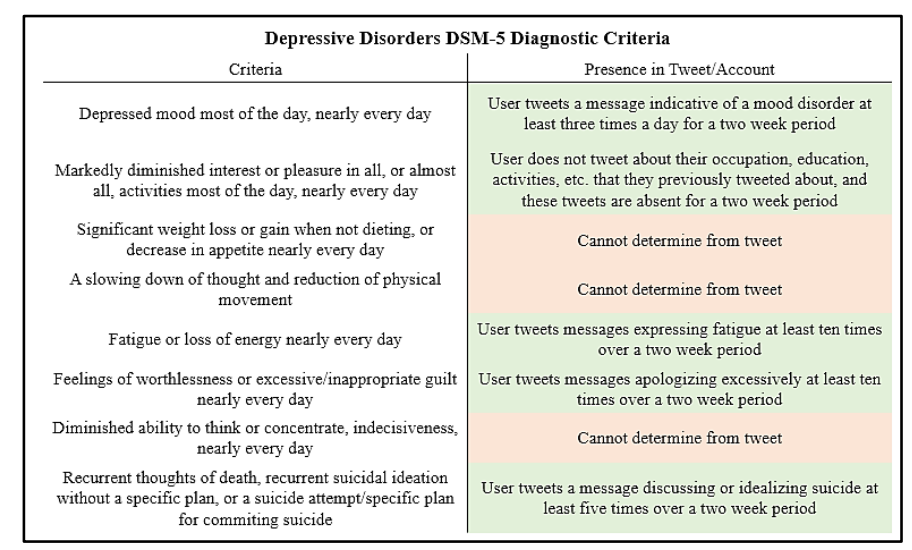

Using the identified individuals, a second Python script utilizing Twitter's API was developed to collect all past tweets from the users' timeline. If the user had less than 10 tweets in their timeline, they were not included, and a maximum of 100 tweets were used from each user. The two datasets (from presumably depressed users and from healthy users) were manually filtered again and the train/test datasets were narrowed further to create a reliable set of ground truth data. Additionally, features like gender and age were kept primarily balanced. After this final round of validation, the dataset consisted of 73,944 tweets. Case tweets (31,030 tweets) were labelled with ' 1 ' and control tweets were labelled with ' 0 ' (42,914 tweets). Sample tweets and their corresponding label are displayed below:

\section{Exhibiting a mood disorder:}

"was about to recommend some books and then was like did i even really like them? do i even really like anything?"

"Every day, it feels like the whole world hates me"

"No one seems to notice when you're down unless you are bleeding."

\section{Exhibiting a healthy emotional state:}

"my literally best friend for years is coming to UGA this weekend and I CANNOT WAIT to show her how to be a dawggggg"

"Happy Birthday to me, feels great to be 22. Going to be a big and great year for me."

\section{Pre-processing}

Once the twitter data was read in to Python and sorted into case and control cohorts, pre-processing was conducted. All Unicode characters and emoticons were replaced with an empty string to ensure that these special characters/symbols did not contribute to model training. The dataset was split into train $(80 \%)$ and test $(20 \%)$ groups and the train data was split into 4 folds. The folds allow for cross-validation in model training. Test data is in fold 0 as seen in the following table:

\section{Linguistic Analysis}

Analysis of linguistic structure and nature was conducted on the entire dataset (both train and test). Two structural patterns were measured: use of indefinite pronouns ("everything", "anybody", "no one") and use of singular first-person pronouns ("myself", "own"). Two topic-based patterns were measured: indication of drug use ("smoking", "alcohol") and indication of outward insecurity ("ugly", "fat"). Odds ratio and relative risk were both computed for each pattern.

\section{Model Training}

In order to determine the specific biomarkers indicative of mood disorders, the tweets were divided into unigrams and bigrams, which were stored in a vocabulary. However, not all words in the original vocabulary were considered features in model training. Only words that exist in a specific percentage $(0.1 \%)$ of tweets were included in the final vocabulary, so each feature (unigram/bigram) is present in at least $\sim 70$ tweets. A training matrix with a row corresponding to each training document and a column corresponding to each biomarker/n-gram was created. A test matrix was also created following the same format. If the n-gram is present 
in the document, a 1 is placed in the cell. All other cells are set to 0 and stored in a sparse matrix format. A column with the classification of the tweet ( 0 for healthy; 1 for mood disorder) was added as the dependent variable. The Generalized Linear lasso Model hyperplane-based approach) from the $\mathrm{H} 2 \mathrm{O}$ Library and 4-fold cross validation were used to train the first model. Next, a tree-based approach (Gradient Boosting Machine) was tried. Lastly, a neural network-based approach (Multilayer Perceptron) was built. After the models were created, a completely new set of documents (test matrix) were scored.

\section{App Design}

iSense was designed to detect a possible mood disorder by implementing the logistic regression model. The app, created in Android Studio, scores all outgoing messages from a teen's phone and stores the score in Google's Firebase Realtime Database. This ensures that the message itself does not leave the phone, hence protecting security. After both the adult and minor agree to the terms of service, relevant statistics are computed and stored. Parents, whose accounts are linked with their teen, can view these statistics in real-time. They can also receive push notifications and set a score threshold; if an outgoing message has a score higher than this threshold, an alert will be sent. The child interface contains a chatbot utilizing natural language processing to stimulate conversation. Much like a teen is likely to be open to a friend over SMS, they are likely to reveal how they feel to a chatbot that does not have the ability to pass judgement.

\section{Results - Linguistic Analysis}

As seen in the Figure 1, all four of the tested linguistic patterns had significant odds ratios. Outward insecurities including signs of body dysmorphia, eating disorders, and low selfconfidence had the highest odds ratio of 10.515. An enlarged use of generalized and negative indefinite pronouns was also seen, with an odds ratio of 3.315 . Language that indicated drug use or withdrawal symptoms had an odds ratio of 3.297. Singular firstperson-pronouns and other self-referent word usage had an odds ratio of 2.139. All odds ratios were greater than one, indicating that if these linguistic biomarkers exist, the subject is more likely to have a mood disorder.

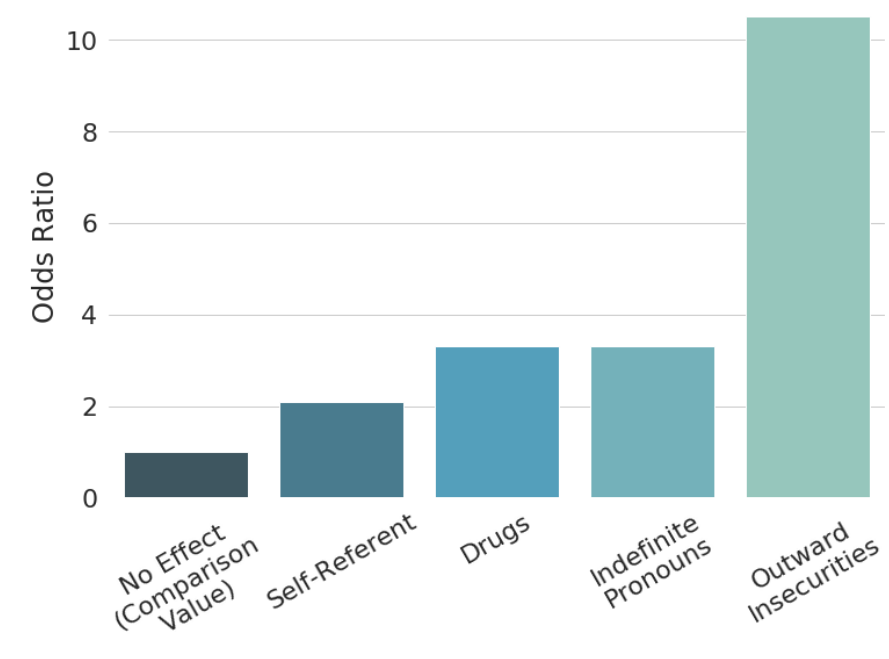

Figure 1

Linguistic Structure/Nature

Figure 2 displays the relative risk for the same linguistic patterns described above. Relative risk provides an accurate representation of the relationship between exposure and outcome when the prevalence of the case is high, like with mood disorders. All of the risk ratios were greater than one (the comparison value) and hence indicate the risk of a mood disorder is higher if these linguistic patterns are present. Outward insecurities, language that indicates drug usage, indefinite pronouns, and selfreferent word usage had relative risks of 2.108, 1.685, 1.677 , and 1.453 , respectively.

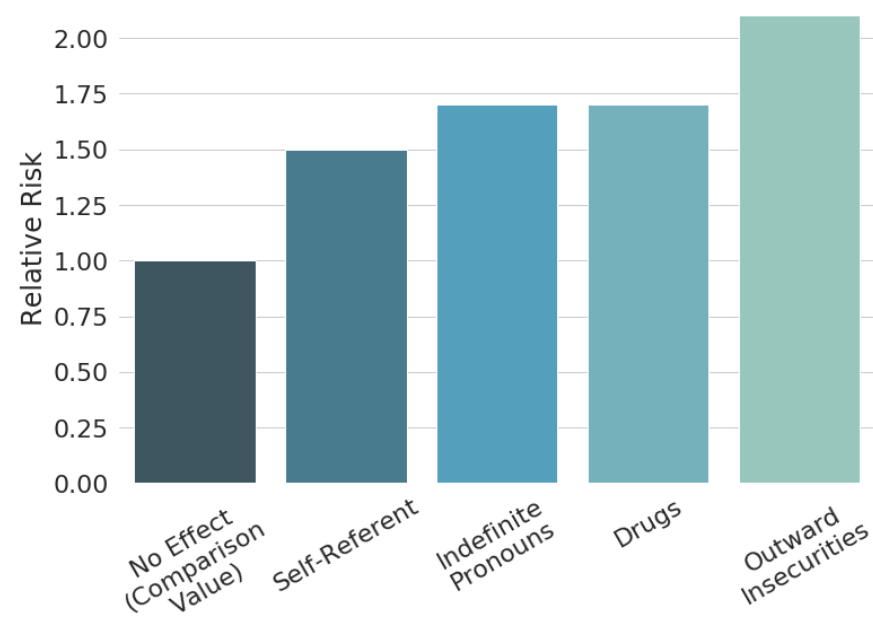

Figure 2 


\section{Results - Generalized Linear Model}

The Receiver Operating Characteristic (ROC) curve (Figure 3) plots the False Positive Rate (1 specificity) against the True Positive Rate (sensitivity). The area under the curve (AUC) is a measure of how well the model can distinguish between cases and controls, with the diagonal representing a random sort. The closer the curve comes to the 45-degree diagonal, the less accurate the model. Model training had an AUC of 0.7892 and model testing had an AUC of 0.7860. Since the difference between the two values is low, the model likely did not overfit to the training data. The Precision-Recall Curve (Figure 4) plots Recall (sensitivity) against Precision (positive predictive recall). These curves are useful in situations in which the positive class is of higher than interest than the negative class, as in this study. 0.7388 is the average precision across all sensitivities in test.

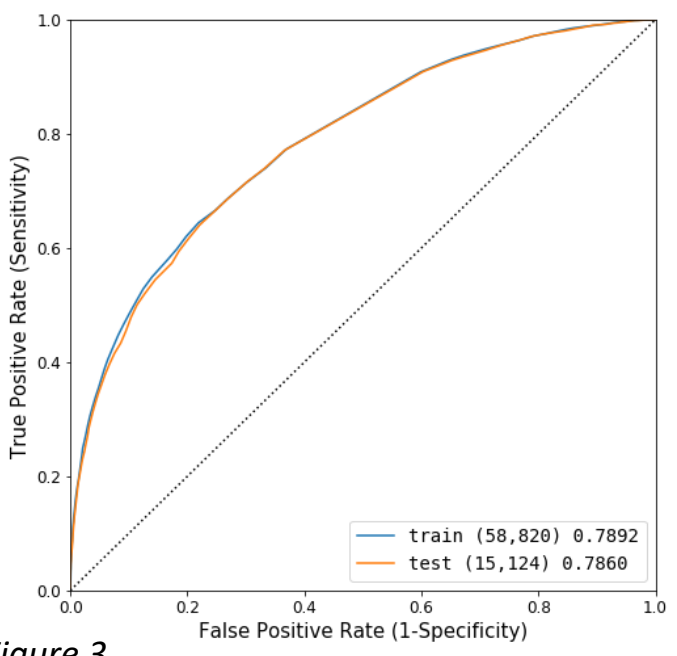

Figure 3

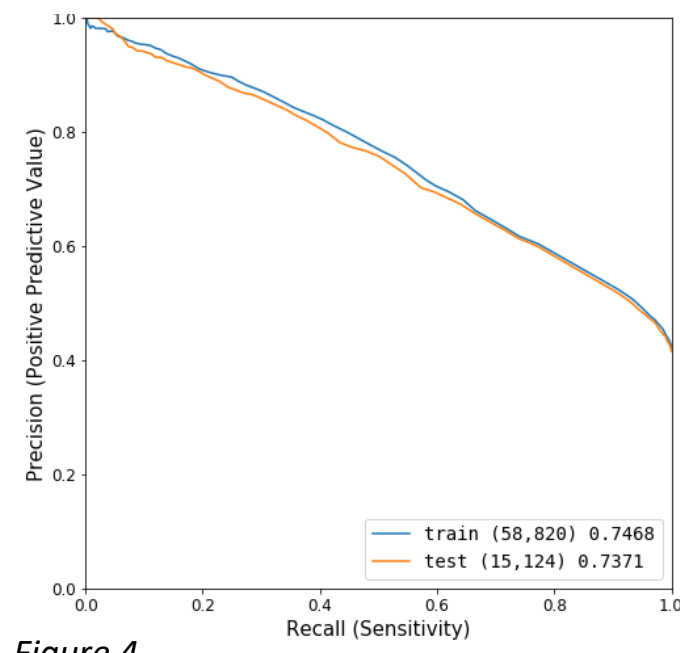

Figure 4
Figure 5 shows the number of documents in each score bin by percentage of observations per class. The score bins with solely dark blue bars were always classified as cases, the score bins with solely yellow bars were always classified as controls, and the brown bars indicate the intersection between cases and controls. In model training/testing, the mean score of the cases was 0.51 and the standard deviation was 0.16. The mean score of the controls was 0.36 and the standard deviation was 0.11 .

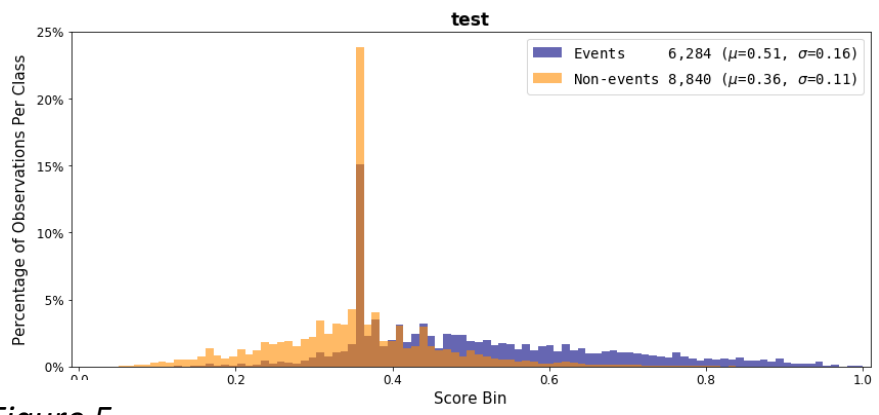

Figure 5

Figure 6 shows accuracy, sensitivity (true positive rate), and specificity (true negative rate) as the score threshold between case and control changes. In order to determine the optimal threshold, these measures need to be maximized. In training, 0.46 was the score threshold where accuracy is maximized and 0.38 was the score threshold where all three measures are optimal.

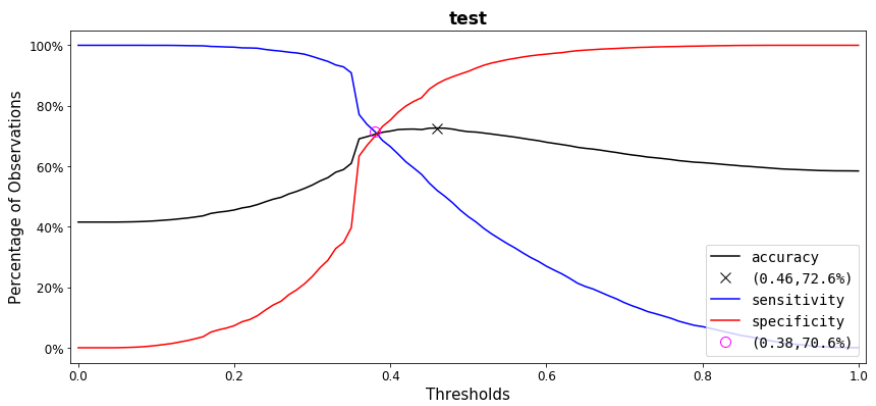

Figure 6

\section{Important Linguistic Biomarkers}

The following linguistic biomarkers (Figure 7) were determined to be the most predictive of mood disorders. The intercept value was -0.672 . The most predictive word was "pain" with a coefficient of 1.40. 


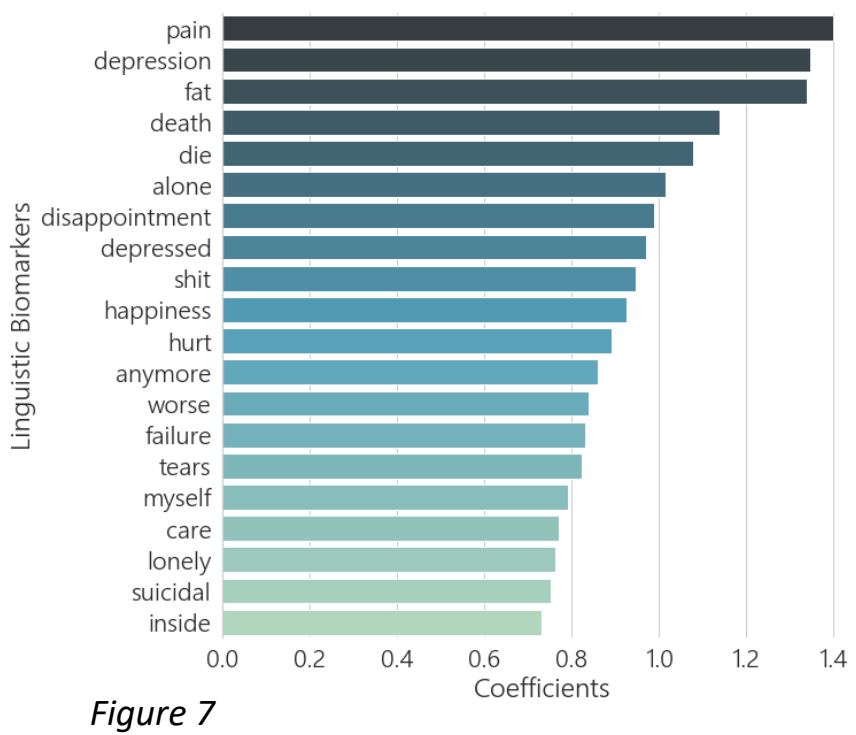

Results - Gradient Boosting Machine

Figure 8 shows that model training had an AUC of 0.8213 and model testing had an AUC of 0.8092, indicating that the model did not overfit to the training data. Figure 9 shows that 0.7665 is the average precision across all sensitivities in test. Figure 10 shows the linguistic biomarkers with the highest variable importance.

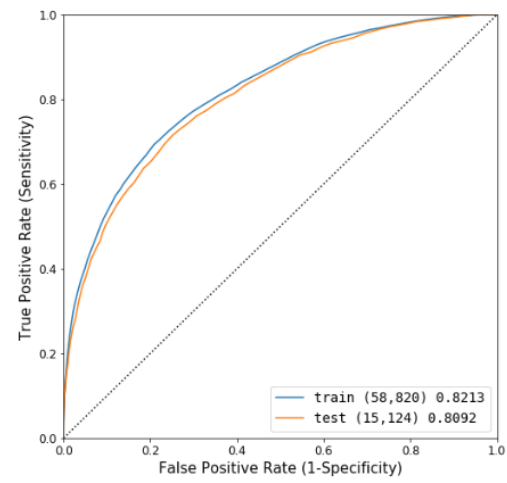

Figure 8

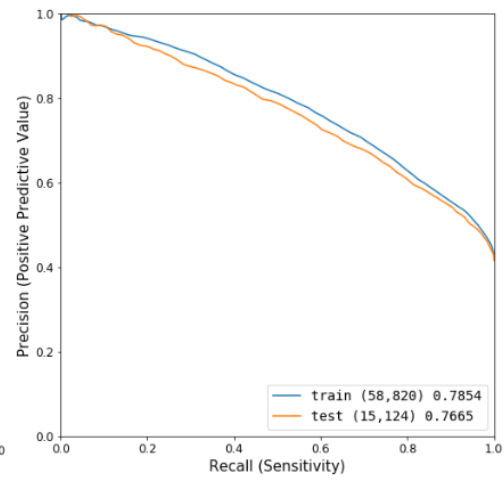

Figure 9

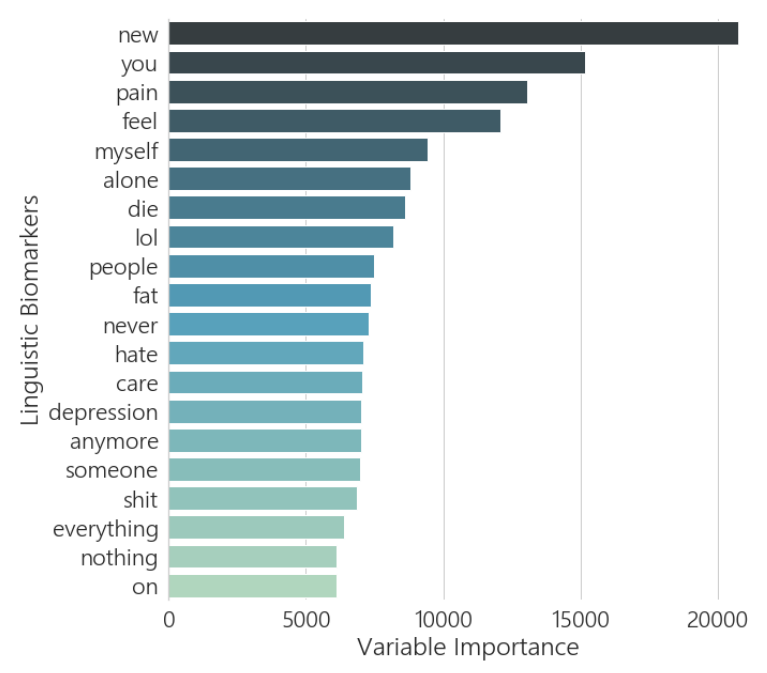

Figure 10
As shown in Figure 11, in model training/testing, the mean score of the cases was 0.54 and the standard deviation was 0.19 . The mean score of the controls was 0.33 and the standard deviation was 0.14 . Figure 12 shows that in testing, 0.48 was the score threshold where accuracy is maximized, and 0.40 was the score threshold where all three measures are optimal

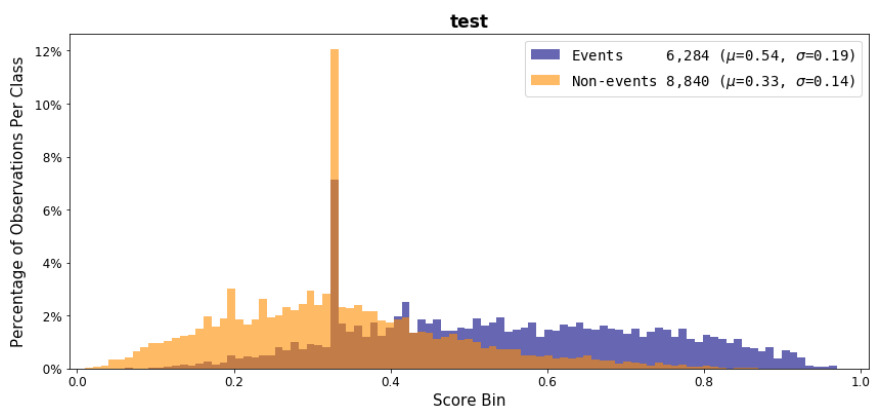

Figure 11

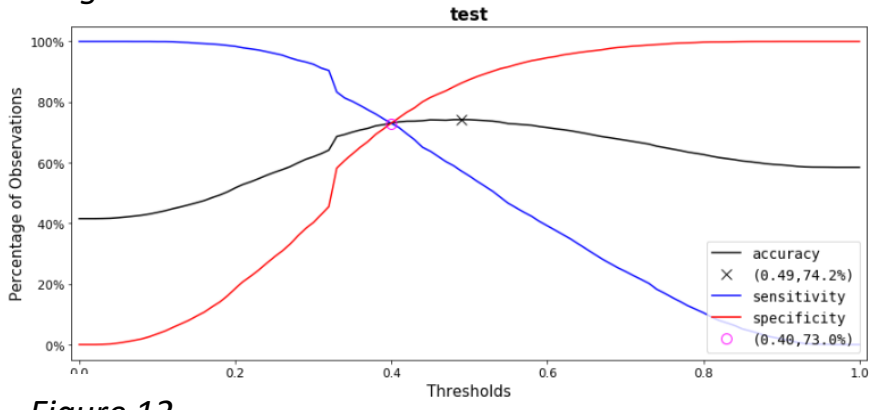

Figure 12

\section{Results - Neural Network}

Figure 13 shows the architecture of the feed-forward deep neural network: an input layer, an output layer, and two hidden layers. 991 features (linguistic biomarkers) are in the input of the first layer. 52,201 total parameters are computed between all layers, and one score is returned. The Rectified Linear Unit (ReLU) activation function is used for the hidden layers, and the Sigmoid activation function is used for the output layer

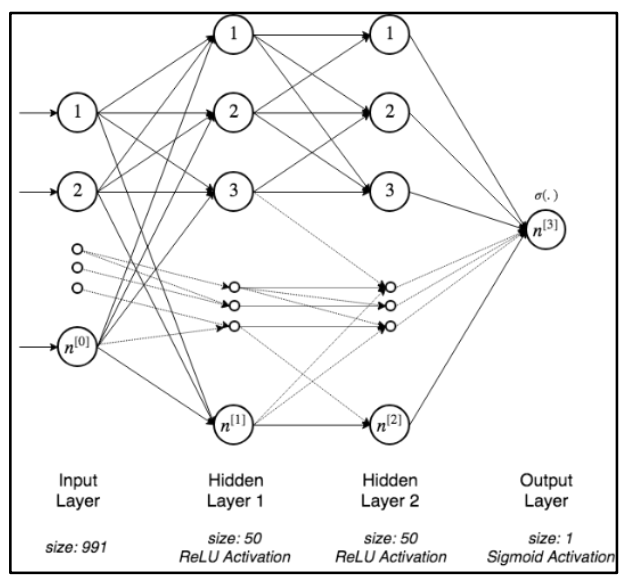

Figure 13 
Figure 14 shows that model training had an AUC of 0.8570 and testing had an AUC of 0.8373. Figure 15 shows that 0.8006 is the average precision in testing.

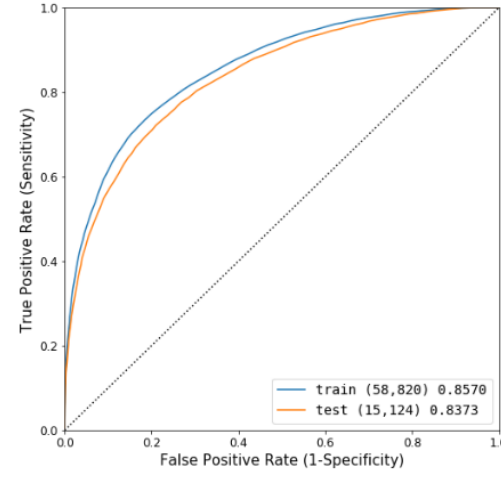

Figure 14

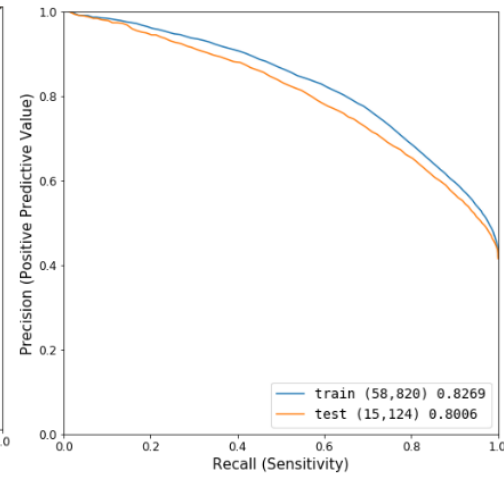

Figure 15
As shown in Figure 16, in model testing, the mean score of the cases/controls were $0.59 / 0.30$ and the standard deviation was $0.23 / 0.18$ respectively. Figure 17 shows that in testing, 0.46 was the score threshold where accuracy is maximized, and a threshold of 0.38 optimizes all measures.

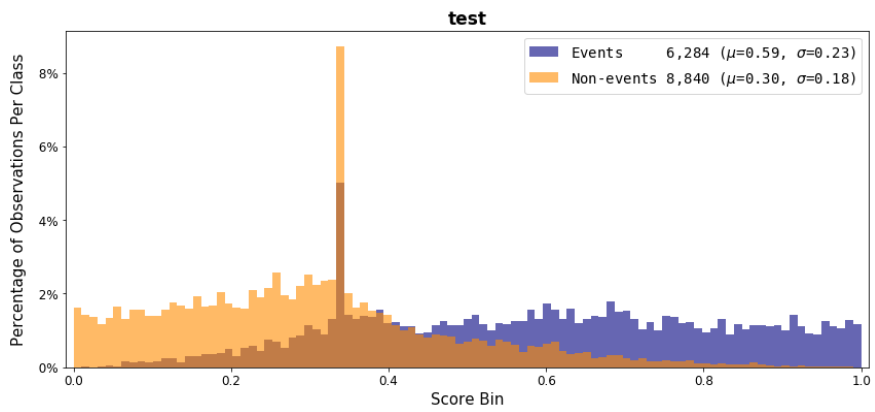

Figure 16

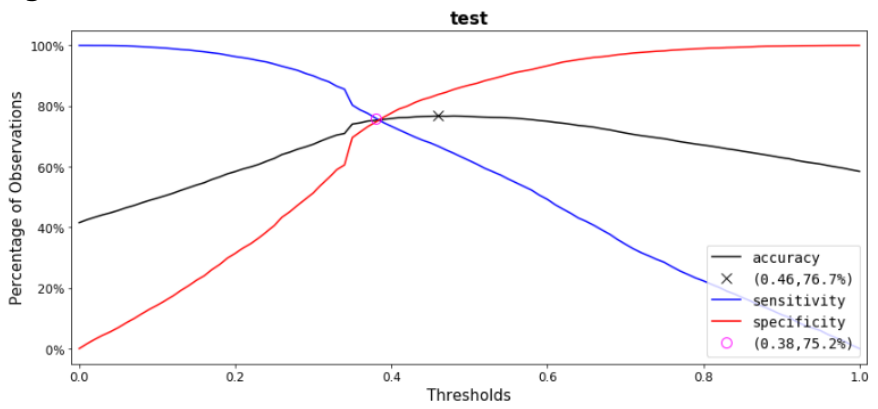

Figure 17

\section{Shapley Values}

Shapley values, a method originating from game theory, helps quantify the contribution of each feature in a prediction. The original problem is, in a team that is composed of multiple players with differing skill sets, that results in some collective payoff, what is the fairest way to divide up that payoff among the players? The Shapley value is the average marginal contribution of a feature value across all possible feature combinations or coalitions.

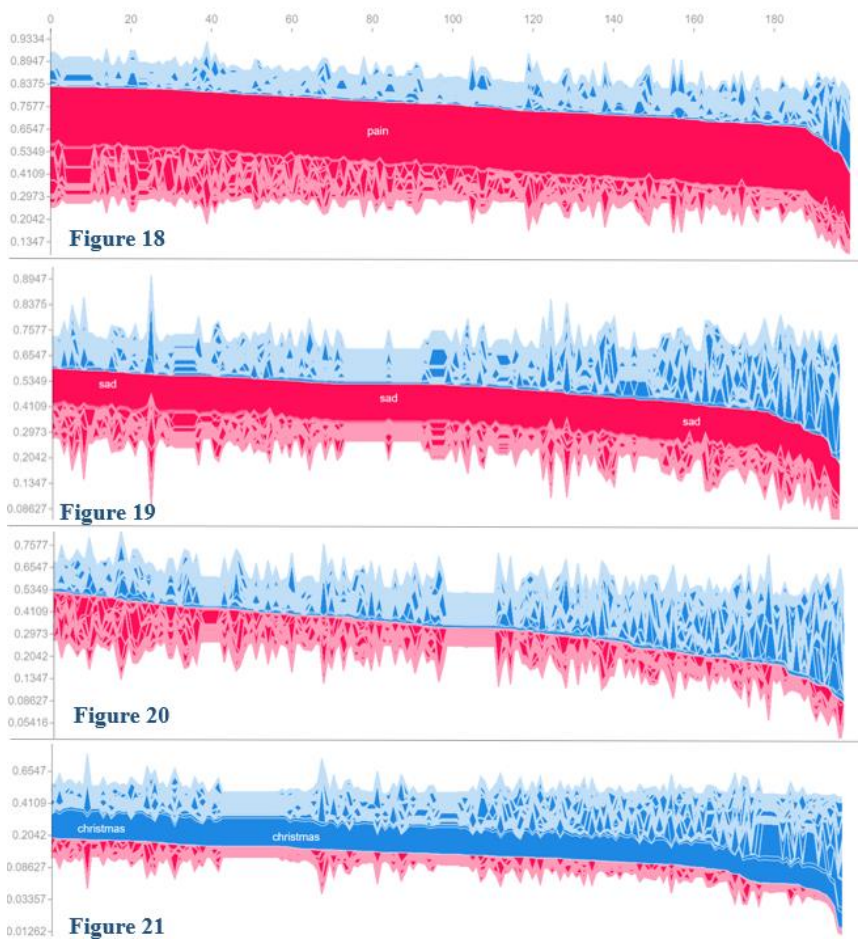

Figures 10-13 show that while tweets with "pain" and "sad" are almost always predicted as cases, tweets with "family" can be cases or controls, depending on other words used, and tweets with "Christmas" are almost always controls. Figures 14 and 15 show Shapley values of words in two example tweets (impact of the word on the score is indicated by the length of the word's bar)

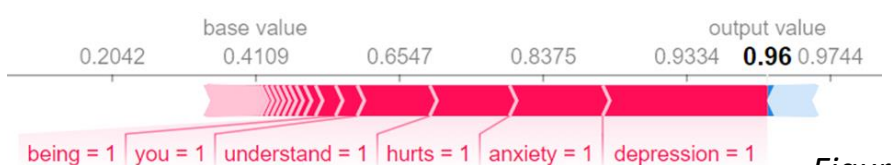

Figure 14

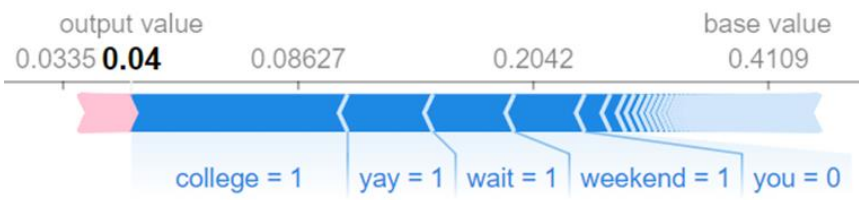

Figure 15

\section{Conclusions}

The hypothesis was supported in that there exists a measurable difference in the language use of an individual with a mood disorder when compared to a 
healthy individual's language use. 0.4 was determined to be the threshold because F1 Score is maximized at this value. These conclusions were implemented in iSense, a mobile app that has the ability to to effectively and efficiently detect mood disorders.

\section{Future Work}

Future directions could include analyzing data from other social media sources to obtain a wider sample and a larger vocabulary. The next iterations of the existing model could be trained to distinguish between existence of depression as part of a unipolar or bipolar disorder. An iOS version of iSense could be created to allow for universal use, and an increased number of app features could be added to improve its effectiveness.

\section{Literature Cited}

[1] - "Any Mood Disorder." National Institute of Mental Health, U.S. Department of Health and Human Services, www.nimh.nih.gov/health/statistics/any-mooddisorder.shtml\#part_155961.

[2] - Minnesota Department of Health. "Programs \& Initiatives in Schools - Suicide Prevention Minnesota Department of Health.” Airborne Precautions - Minnesota Dept. of Health, www.health.state.mn.us/divs/healthimprovement/pr ograms-initiatives/in-schools/preventsuicide.html.

[3] - Brockmeyer, et al. "Me, Myself, and I: SelfReferent Word Use as an Indicator of Self-Focused Attention in Relation to Depression and Anxiety." Frontiers, Frontiers, 28 Sept. 2015, www.frontiersin.org/articles/10.3389/fpsyg.2015.01 564/full.

[4] - "Depression (Major Depressive Disorder)." Mayo Clinic, Mayo Foundation for Medical Education and Research, 3 Feb. 2018, www.mayoclinic.org/diseasesconditions/depression/symptoms-causes/syc20356007?utm_source=Google\&utm_medium=abst ract\&utm_content=Majordepression\&utm_campaign=Knowledge-panel.
[5] - "National Center for Biotechnology Information." Current Neurology and Neuroscience Reports., U.S. National Library of Medicine, www.ncbi.nlm.nih.gov-PMC-5902561/.

[6] - "National Center for Biotechnology Information." Current Neurology and Neuroscience Reports., U.S. National Library of Medicine, www.ncbi.nlm.nih.gov-PMC-5964306/.

[7] - "How to Differentiate Bipolar Disorder from Unipolar Depression.” Cyberbullying Linked With Range of Mental Health Effects, www.psychcongress.com/article/how-differentiatebipolar-disorder-unipolar-depression.

[8]- "Mood Disorders." Mayo Clinic, Mayo Foundation for Medical Education and Research, 20 Dec. 2018, www.mayoclinic.org/diseasesconditions/mood-disorders/symptoms-causes/syc20365057.

\section{Programming Reference}

"H2O.Ai Documentation." Generalized Linear Model (GLM) - H2O 3.20.0.8 Documentation, docs.h2o.ai/.

"Firebase Realtime Database | Firebase Realtime Database | Firebase." Google, Google, firebase.google.com/docs/database/.

"Natural Language Processing with Python." NLTK Book, www.nltk.org/book/. 